\title{
ㄷำ1
}

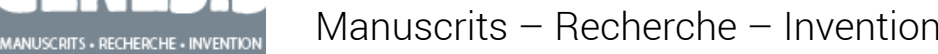

43 | 2016

Bande dessinée

\section{Planches originales : préserver, restaurer, lire}

Entretien avec Jean-Pierre Mercier

Luc Vigier

\section{(2) OpenEdition}

Journals

Édition électronique

URL : http://journals.openedition.org/genesis/1708

DOI : $10.4000 /$ genesis. 1708

ISSN : 2268-1590

Éditeur :

Presses universitaires de Paris Sorbonne (PUPS), Société internationale de génétique artistique littéraire et scientifique (SIGALES)

\section{Édition imprimée}

Date de publication : 12 décembre 2016

ISBN : 9791023105490

ISSN : $1167-5101$

\section{Référence électronique}

Luc Vigier, «Planches originales : préserver, restaurer, lire », Genesis [En ligne], 43 | 2016, mis en ligne le 29 novembre 2017, consulté le 19 avril 2019. URL : http://journals.openedition.org/genesis/1708 ;

DOI : 10.4000/genesis.1708 


\title{
Planches originales : préserver, restaurer, lire
} Entretien avec Jean-Pierre Mercier

\author{
Propos recueillis par Luc Vigier
}

\begin{abstract}
Jean-Pierre Mercier est aujourd'hui conseiller scientifique du musée de la Bande dessinée à Angoulême et vient d'organiser une exposition importante des originaux de Morris, créateur de Lucky Luke. Immergé très tôt dans l'univers du fanzinat (il collabore à Falatoff), il devient bibliothécaire à la Ville de Paris et se passionne pour les albums destinés à la jeunesse où rayonnent alors des artistes comme Ungerer, Sendak, Lobel. Le lien s'effectue assez naturellement ensuite entre l'illustration et la bande dessinée, dont il devient progressivement - par recherches, enquêtes, traductions et interviews, à une époque où peu de données scientifiques existent sur le neuvième art - un spécialiste enthousiaste et précis, tout en développant des activités d'éditeur de bande dessinée au sein des éditions Artefact. Responsable de nombreuses expositions dont il a coordonné les catalogues, pédagogue infatigable, Jean-Pierre Mercier valorise un patrimoine unique en Europe. Il a bien voulu nous recevoir à l'occasion de ce numéro de Genesis pour évoquer l'histoire du fonds de manuscrits et de dossiers graphiques patiemment constitué depuis près de trente ans.
\end{abstract}

Musée de la Bande dessinée, Angoulême, le 22 octobre 2015

Luc Vigier - Pouvez-vous revenir sur vos débuts au musée de la Bande dessinée d'Angoulême?

Jean-Pierre Mercier - Nous sommes en 1988. Je me dis que je vais rester trois ou quatre ans et puis revenir à Paris pour entreprendre autre chose. Cela fait vingt-six ans que je suis angoumoisin... J'arrive sur place pour monter un équipement, il faut recruter, constituer les collections, réfléchir à un système de classement spécifique. Par chance, on dispose du dépôt légal BD. En 1984, la bibliothèque municipale d'Angoulême a signé une convention avec la Bibliothèque nationale : un exemplaire de tout ce qui paraît en, ou sur la bande dessinée est envoyé à Angoulême. Nous récupérons ce dépôt avec mission de le traiter. Cela représentait des dizaines de cartons. Par ailleurs, le directeur de l'époque avait été contacté par Eudes de la Potterie, du centre de documentation sur la presse jeunesse créé en 1946 par les éditions Fleurus, qui venait de fermer. Ce monsieur cherchait un point de chute pour l'énorme collection accumulée. Et c'est ainsi que nous avons récupéré d'autres dizaines de cartons. Sur cette belle base, nous avons commencé un travail de constitution d'archives qui se poursuit toujours aujourd'hui. Nous suivons les parutions et complétons les collections anciennes. Le catalogue est en ligne et compte des dizaines de milliers de références. Au bout de trois ans, il y a eu des remaniements dans la maison, et on m'a proposé de quitter la direction de la bibliothèque pour devenir ce que je suis encore aujourd'hui, conseiller scientifique. Il fallait renforcer les compétences en matière de contenu. J'ai accepté immédiatement. Je suis donc désormais une des personnes ressources de l'établissement, je travaille aussi bien avec la bibliothèque, le musée, le service communication, je prends en charge le commissariat des expositions, la rédaction d'articles, des formations de bibliothécaires, d'enseignants, de formateurs. En ce moment, je m'occupe de la grande rétrospective consacrée à Lucky Luke. Mon travail habituel consiste à participer à la constitution des collections, à suivre les ventes aux enchères, éplucher les listes de ventes de libraires spécialisés, répondre aux demandes un peu pointues des journalistes, de certains chercheurs ou des simples curieux, d'établir des contacts avec les auteurs, les institutions comparables à la nôtre à l'étranger, proposer des projets d'expositions... C'est très varié, et très prenant.

L.V. - À quel moment se fait l'arrivée massive des originaux?

J.-P. M. - Dans nos collections? Avant même l'existence du musée. Le Festival de la bande dessinée d'Angoulême démarre en 1974, et le conservateur du musée municipal d'Angoulême de l'époque, dont le nom m'échappe hélas, 
a un réflexe merveilleux : il demande à chaque auteur qu'il croise au moment du Festival : «Ça vous ennuie de nous laisser une planche?» Il constitue ainsi une collection de peut-être cinq cents planches que nous récupérons ensuite. Il y a de très belles choses, dont une double page du Sceptre d'Ottokar, une planche crayonnée et une planche encrée de Tintin au Tibet, qui ont été achetées à Hergé. Cette collection est une nouveauté pour l'époque, c'est un très beau musée d'art graphique qui comprend aujourd'hui treize mille planches. Ce sont des documents fragiles qui nécessitent des conditions d'exposition très contraignantes : un mois d'exposition pour un an de repli dans les réserves, une atmosphère à $18^{\circ} \mathrm{C}$ de température, avec $50 \%$ d'humidité relative et, quand un document est exposé, une lumière de 50 lux maximum.

\section{L.V. - Le fonds n'est pas constitué uniquement des dons de dessinateurs?}

J.-P. M. - Non, bien entendu. Nous avons un budget spécifique pour les achats, qui sont de toute nature : les artistes eux-mêmes, les héritiers à leur mort, les collectionneurs, les maisons de vente, les galeries spécialisées qui naissent dans les années quatre-vingt et quatre-vingt-dix...

L.V. - Des étonnements pour vous dans la découverte des archives et des étapes antérieures à la publication?

J.-P. M. - J'ai été éditeur, je savais ce qu'est une planche de bande dessinée, un scénario, des esquisses, un bleu de mise en couleur, un ozalid, un mash-print, etc. Je me suis formé de façon plus fine sur ce qu'on appelle les «matières et techniques » : le papier, les calques, les rhodoïds, je connais. Je reconnais l'aquarelle, la gouache, mais je suis très prudent sur tout le reste. Les premières années on achète et on traite des planches, mais très vite on se dit que les étapes de conception - le premier synopsis, le découpage, les croquis, les recherches -, c'est passionnant et ça renseigne le processus de création.

\section{L.V. - Ce processus commence par le texte?}

J.-P. M. - La plupart du temps, oui, d'une manière ou d'une autre, mais il existe des auteurs qui se lancent directement,

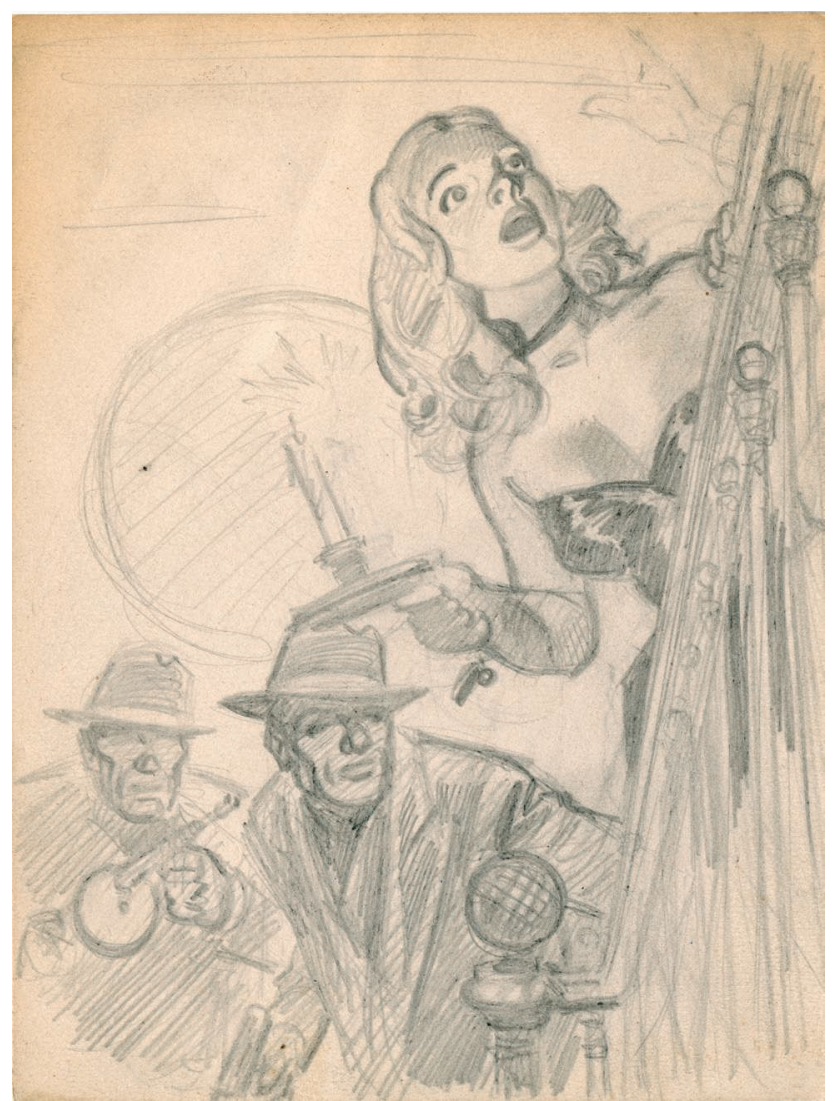

Fig. 1 : Raymond Poïvet, esquisse pour l'illustration de couverture d'un roman policier, années 1950 (C) Raymond Poïvet (Collection musée de la Bande dessinée, Angoulême)

Robert Crumb par exemple, pour ses histoires courtes. Il a une idée, puis il se lance directement dans le dessin. D'autres au contraire vont tout écrire. Fred, le regretté dessinateur de Philémon, était connu pour avancer de façon non linéaire dans son récit. Il pouvait sauter dix pages, faire une séquence puis revenir en arrière, au gré des envies et de l'inspiration. Des auteurs font des découpages très précis, d'autres posent les silhouettes très schématiquement. Gotlib, héros de ma jeunesse, par exemple, ses crayonnés sont exactement comme ses planches originales terminées : il est dans le contrôle absolu de son trait. À l'inverse, Morris posait un croquis très simple et attaquait directement au pinceau. Il y a le cas courant du binôme scénaristedessinateur. Le cas plus rare du travail à quatre mains : 


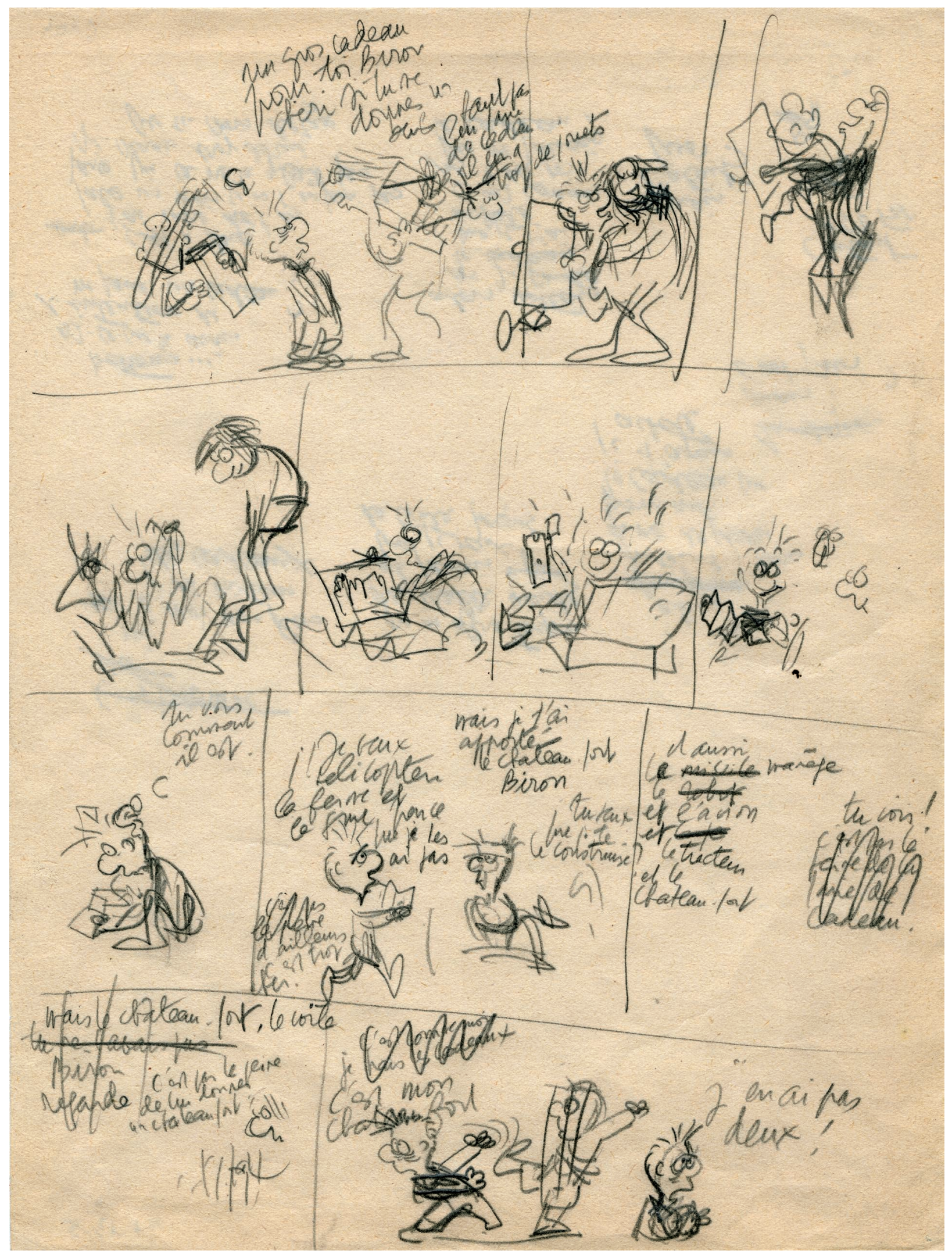

Fig. 2 : Claire Bretécher, découpage crayonné d’une planche d'Agrippine, «Pourboires», années 1990 ๑ Claire Bretécher (Collection musée de la Bande dessinée, Angoulême) 


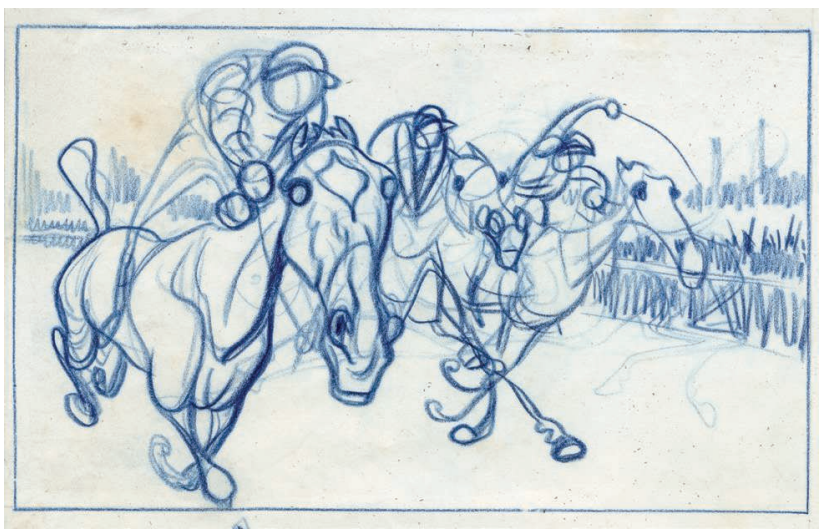

Fig. 3 : Caran d'Ache, esquisse au crayon d'une illustration parue dans la presse, deuxième moitié du XIX ${ }^{\mathrm{e}}$ siècle (Collection musée de la Bande dessinée, Angoulême)

il y a eu Dupuy-Berberian, l'un commençait le dessin l'autre le terminait, indifféremment. Sur le scénario, ils discutaient, prenaient des notes chacun de leur côté, une idée venait de l'un, l'autre la reprenait... On connaît aujourd'hui Ruppert et Mulot. Le coloriste est de plus en plus souvent reconnu dans le travail de création : on peut citer Isabelle Merlet, Brigitte Findakly par exemple. Les auteurs les choisissent pour leur univers, pour leur sensibilité, leur technique, leur vision particulière : ils sont parfois mentionnés comme coauteurs sur les pages de titre.

\section{L.V. - On voit les évolutions des supports dans le temps?}

J.-P. M. - Pour les originaux du tournant des $\mathrm{XIX}^{\mathrm{e}}-\mathrm{XX}^{\mathrm{e}}$ siècles, les formats sont souvent très grands, sur des papiers assez solides, du vrai beau papier à dessin - je pense aux Américains Outcault ou bien sûr McCay, les grands formats. Ça se diversifie, puis on suit l'arrivée de la trame mécanique, du Double Tone... Ensuite les grandes maisons américaines DC et Marvel instituent un système de production qu' on peut qualifier d'industriel : le travail de création est divisé entre un crayonneur, un encreur, un lettreur, un coloriste. L'éditeur fournit à ses auteurs des planches préimprimées en bleu clair qui indiquent la surface utile du dessin, délimite les cases, la zone de titre... Il y a en haut une zone «technique» pour l'éditeur où sont inscrits les numéros de page, le titre de l'épisode, le numéro de la revue, etc.
L.V. - Une anticipation de l'organisation visuelle de la vignette...

J.-P. M. - Exactement. Avec les comics américains, on est clairement dans un processus industriel. C'est différent de l'Europe où l'auteur est libre de ses choix en matière de support, de format, d'outils. Morris travaillait sur de très grands formats, Trondheim a longtemps dessiné sur des feuilles d'imprimante.

L.V. - Est-ce qu'on a assez de pièces pour comprendre ce qui s'est passé?

J.-P. M. - C'est une préoccupation récente. Il ne faut pas oublier que l'étude de l'histoire de la bande dessinée à moins de cinquante ans. Il y a une dizaine d'années, un particulier nous a apporté deux grosses valises remplies de grands dossiers, des valises remplies de documents liés à la fabrication d'images d'Épinal, provenant de la maison parisienne Quantin, producteur tardif de cette imagerie populaire. Quantin voulait faire de l'image artistique dans un domaine qui ne brillait pas par son ambition de ce côté. Il recrute Job, Caran d'Ache, Rabier et des tas de peintres oubliés aujourd'hui. C'est un peu la fin de la vogue de cette production populaire qui va péricliter après la guerre de 1914. Il y a dans ce fonds environ trois cents dossiers comprenant, quand ils sont complets, la page originale, le texte manuscrit, les placards typographiés avec corrections, la mise en couleurs à l'aquarelle de l'auteur, la mise en couleurs aux pochoirs, avec des nuances, les épreuves de tirage et le produit fini... C'est très instructif pour expliquer les étapes de fabrication. Certains procédés ont changé, mais le déroulement général de la fabrication reste le même.

L.V. - Dans les années soixante-dix, est-ce que la notion d'art domine l'idée de la bande dessinée?

J.-P. M. - Elle commence à émerger. Il y a des tentatives périphériques de bandes dessinées qui s'inspirent du nouveau roman, par exemple, qu'on redécouvre aujourd'hui. Dans le champ spécifique de la création $\mathrm{BD}$, des auteurs et quelques critiques commencent à faire valoir que la bande dessinée est une forme artistique aussi valable que toutes les autres. Les auteurs qui ont connu les périodes antérieures sont 


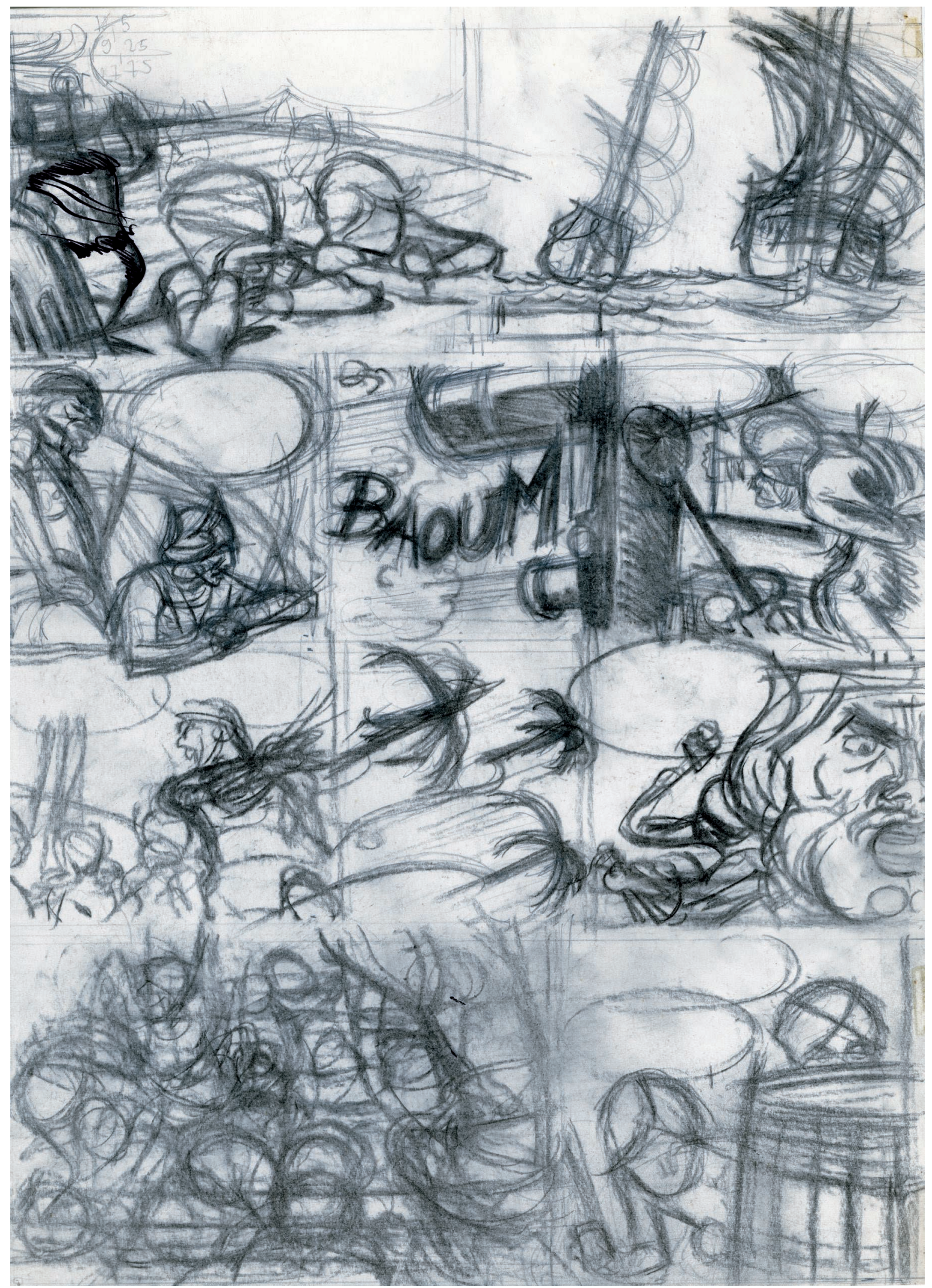

Fig. 4 : Paul Cuvelier, esquisse au crayon de la planche 8 de Corentin, Le Poignard magique, 1963 @ Paul Cuvelier (Collection musée de la Bande dessinée, Angoulême) 
souvent mal à l'aise avec le statut d'artiste et beaucoup le récusent. Aujourd'hui, on peut être auteur de bande dessinée et se revendiquer artiste sans difficulté. L'existence même de notre musée participe, certes marginalement, à la constitution du marché de la planche originale

L.V. - Lors du décès d'un dessinateur, est-ce que vous bénéficiez de dons?

J.-P. M. - Oui, de temps à autre. Depuis quelques années, nous récupérons également des fonds de chercheurs comme Pierre Couperie. Son exécutrice testamentaire nous a fait don de tout ce qu'il avait amassé en documentation BD. C'est énorme. On a reçu des fonds plus modestes de la famille de Jean-Claude Glasser, Annie Baron-Carvais, Jacques Dutrey. Si l'on parle des auteurs, notre plus grande opération est l'achat de mille deux cents originaux d'Edmond-François Calvo. Nous connaissions sa fille, qui avait la responsabilité du patrimoine artistique de son père. Le petit-fils de Calvo nous a contactés pour un dépôt de près de deux mille pièces, qu'on a transformé quelques années plus tard en achat de mille deux cents pièces. Quand on contemple des originaux de Calvo, l'émotion est forte. C'est un grand maître, hélas trop méconnu. J'espère que nous pourrons faire une grande opération de mise en valeur de ce fonds, et de toute l'œuvre de Calvo, qui est majeure. C'est une chance d'avoir toutes ces pièces, conservées dans la famille. Pour d'autres auteurs, tout a été jeté ou détruit. Jusque dans les années soixante, la planche n'a aucune valeur, on marche dessus, on la jette, on déchire, les auteurs autant que les éditeurs et les imprimeurs ! Cela change dans les années soixante-dix et quatre-vingt, quand des auteurs qui connaissent le fonctionnement du marché de l'art décident de monnayer leurs originaux. Le processus est lent et progressif, mais il est désormais bien installé. Certains auteurs, comme Druillet ou Bilal, jouent sur plusieurs tableaux : ils sont auteurs de bande dessinée, peintres, décorateurs pour des films, voire même réalisateurs. Leur travail «multicarte» leur permet d'asseoir une certaine légitimité auprès des collectionneurs.

L.V. - J'imagine que vous utilisez les technologies les plus récentes, notamment la numérisation, justement, pour conserver les originaux?
J.-P. M. - Bien entendu. Notre premier travail est donc de constituer une collection pour établir une mémoire, en faire la conservation et la restauration (nous avons des budgets de restauration annuels) et d'en assurer la mise en valeur au moyen d'expositions, de catalogues et de présentation du musée. On sait très bien qu'il faut préserver tout cela et la préservation passe par le fait qu'on les manipule le moins possible. La numérisation facilite la divulgation et permet de limiter les manipulations. C'est très précieux.

L.V. - Est-ce qu'il y a dans les dossiers des éléments photographiques, des documents visuels préparatoires?

J.-P. M. - Les auteurs sont discrets là-dessus, nous en avons peu. Mais on sait que certains auteurs, et non des moindres, comme Tardi, recourent massivement à la photo. Dans son cas, il s'en sert pour les décors urbains de ses histoires, par exemple quand il travaillait sur les adaptations des polars de Léo Malet, dont chaque roman se déroule dans un arrondissement spécifique de Paris. Il faisait des repérages, comme un cinéaste. Des reportages ont été faits où on le voit prendre des photos de telle façade, de telle rue. Ensuite, il explique toute la cuisine : quand on prend une photo, selon la focale, les verticales sont plus ou moins déformées. Le regardeur l'accepte pour une photo, mais pas dans un dessin. Tardi s'astreint donc à rétablir dans ses dessins les verticales des photographies qu'il a prises. C'est une des spécificités du dessin sur lesquelles les dessinateurs s'expliquent parfois. Ainsi, on sait que les jambes des personnages, et en particulier des pin-ups, sont beaucoup plus longues en dessin que dans la réalité.

L.V. - La question autobiographique, si souvent présente dans les dossiers d'écrivains, semble plus problématique dans les dossiers génétiques de la bande dessinée, où il y a une sorte d'écran, de distance très forte entre la vie de l'écrivain (sauf dans le cas des démarches autobiographiques comme chez Crumb, ou plus récemment chez Fabrice Neaud) et le dossier préparatoire. Va-t-on trouver chez Morris des éléments autobiographiques, comme on trouve des éléments de la vie personnelle chez Hergé?

J.-P. M. - Beaucoup d'auteurs, mais aussi leurs héritiers, font un tri entre ce qui relève d'une certaine forme d'intimité et 


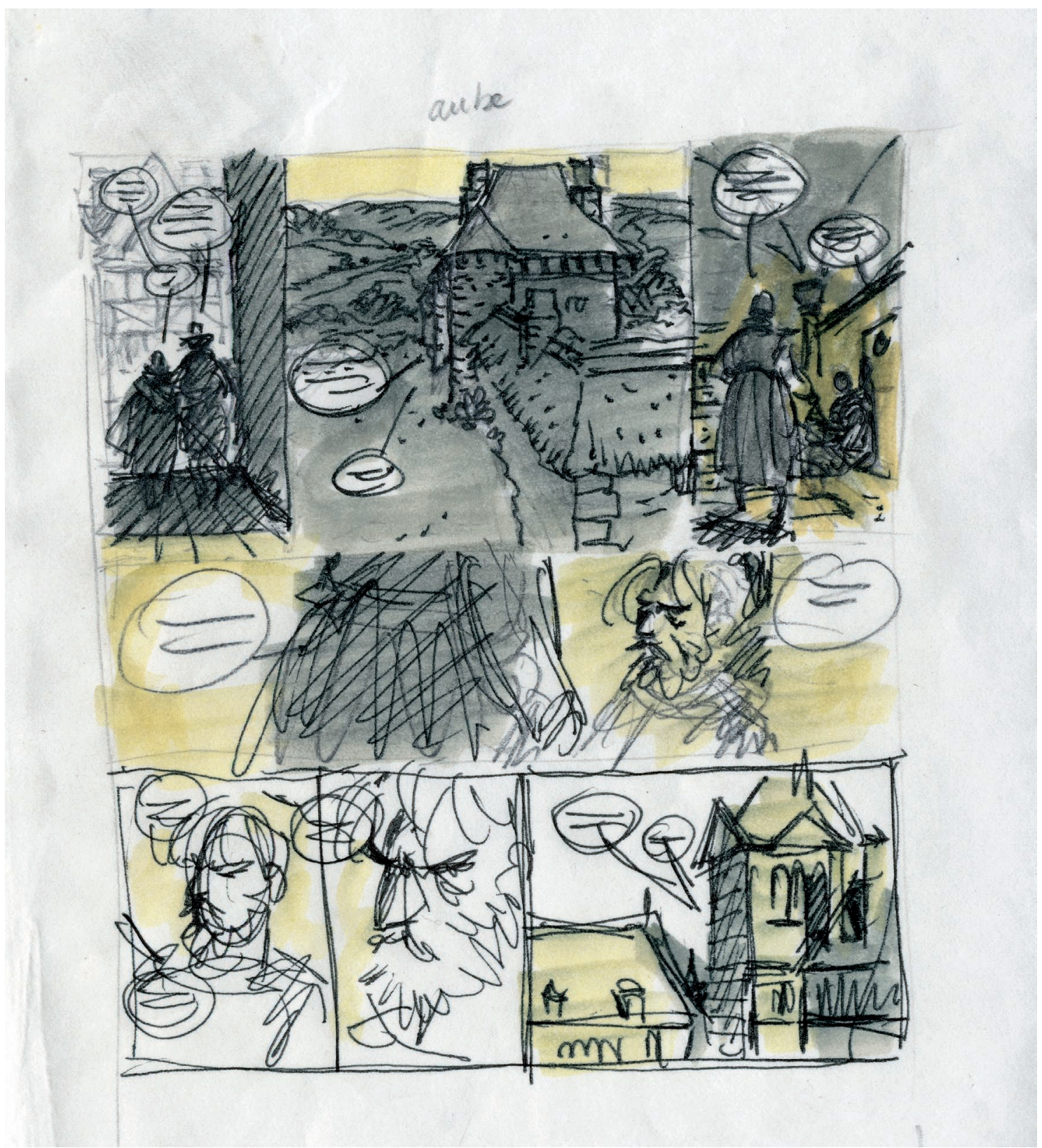

Fig. 5 : André Juillard, découpage crayonné et coloré de la planche 16 des Sept Vies de l'épervier, Hyronimus 1988 @ André Juillard (Collection musée de la Bande dessinée, Angoulême)

de ce qui relève du professionnel. Gilberte Goscinny nous avait donné le dernier scénario de Goscinny pour Astérix et pour Lucky Luke. Nous possédons donc Astérix chez. les Belges et Le Fil qui chante. Dans ce dernier dossier se trouvait une lettre de Morris, qui permet de voir qu'il participait pleinement à la définition du thème des albums qu'il dessinait. Il écrit qu'il a regardé la documentation, qu'il n'y a rien sur l'histoire du télégraphe, puis il enchaîne sur des considérations très prosaïques de technique narrative.
L.V. - Le Fil qui chante, c'est aussi la recherche du trait pur...

J.-P. M. - Le trait pur a été la recherche constante de Morris. Il est parti d'un trait proche du dessin animé, sous l'influence probable de Fleischer et dans une moindre mesure de Disney, puis il est allé vers quelque chose de plus en plus jeté, sous l'influence des auteurs américains - il est resté aux ÉtatsUnis et au Mexique de 1948 à 1954. Il a côtoyé les futurs 
auteurs de Mad comics : Kurtzman, Elder, Wood, et surtout Davis, de mon point de vue sa plus grosse influence.

L.V. - Oui, parce que les premiers Lucky Luke sont un peu «secoués», le mouvement du cheval, assez étrange, et le visage a des allures d'un Elvis Presley survolté...

J.-P. M. - Je pense que l'un des grands horizons de création de cette génération est le dessin animé : Franquin et Morris ont commencé dans le même petit studio de dessin animé, la CBA, qui a rapidement mis la clé sous la porte. Pour les auteurs de cette époque, la référence, l'ambition, c'est le dessin animé. Et pas seulement en Belgique. Pensons au Japon où, juste après la guerre, le jeune Tezuka se rêve en Disney. Il changera de projet et deviendra le maître du manga : il joue l'analogie avec l'expérience de visionnage d'un film et révolutionne le genre.

L.V. - Un dossier d'archives pour lequel vous auriez une préférence?

J.-P. M. - Je vais redire Calvo, c'est une belle histoire. La Bête est morte et Rosalie, qui sont les sommets de son œuvre : La Bête est restée dans la famille de l'auteur, mais Rosalie est désormais dans les collections du musée. Calvo en était très fier et c'est la raison pour laquelle il a fait relier les planches : elles sont collées sur de grands supports en carton pourvu de systèmes de charnières et reliées dans un énorme livre en cuir, qui fait son poids et qui est magnifique. Le problème est que cette reliure a été réalisée juste après Rosalie, en 1947, et l'on voit que près de soixante-dix ans après, l'objet commence à fatiguer. Il y a en particulier une double page centrale qui a été contrecollée sur du carton, ce qui n'est pas recommandé. Elle commence à se fendiller et à se gondoler. Nous allons restaurer cette reliure, mais c'est un gros budget. Sinon, voir des planches que j'ai lues et qui m'ont enchanté quand j'étais enfant, c'est toujours un choc. Travailler au musée m'a guéri du goût de la collection.

\section{L.V. - C'est trop puissant?}

J.-P. M. - J'ai treize mille planches à ma disposition. Je prends la clé, j’ouvre la porte des réserves...

\section{L.V. - L'émotion reste intacte?}

J.-P. M. - Oui, toujours. C'est fascinant, le dessin. Il y a quelque chose de magique. Certains dessinateurs atteignent un tel degré de savoir-faire, de talent, de génie, il y a un effet de bluff, on se fait prendre. Je crois que nous autres pauvres humains, nous avons besoin d'images, de représentation, de signes. Voyez la fascination qu'engendrent la grotte de Lascaux, la grotte Chauvet. Et puis, il y a l'émotion par rapport à l'original, il y a le parfum de l'ancien, du temps passé, le papier jauni, la marque du temps sur le document. C'est un peu mélancolique. Sur l'original, on voit les repentirs, les «rustines », les relectures, une certaine «cuisine» de la création. Il y a des corrections au bleu, à la gouache. J'ai fait deux ou trois interviews de Franquin et on discutait des originaux, des couleurs. Franquin disait, «il ne faut pas oublier que le dessin original préparatoire c'est une matrice, c'est en vue d'une impression. Je pars du principe que je vais perdre entre dix et vingt pour cent de ce que je dessine». Hergé faisait le trait de contour aussi pour que l'on comprenne que si la couleur bave, c'est une question d'impression.

\section{L.V. - Un dernier exemple de découverte?}

J.-P. M. - Nous avons une extraordinaire planche de Mœbius : sur la page originale, une case redessinée a été collée par-dessus la case originale dont il n'était pas satisfait. Sur le dessin original il y a des surfaces noires, traitées à l'encre de Chine, alors que le reste de la planche est uniquement traité en traits de contours, sans aplats noirs. Mœbius est revenu sur cette case par souci d'équilibre de la page entière. 